Case Report

\title{
Localized Chronic Form of Langerhans Cell Histiocytosis in the Femur of a 16-Year-Old Male Successfully Treated with Radiofrequency Ablation
}

\author{
Kyriakos Papavasiliou $\left(\mathbb{D},{ }^{1}\right.$ Antonia Bintoudi ${ }^{(D},{ }^{2}$ Apostolos Vlahodimos, ${ }^{2}$ \\ Eleftherios Tsiridis $\mathbb{D}^{1},{ }^{1}$ Prodromos Hytiroglou ${ }^{(D},{ }^{3}$ Ioannis Tsitouridis, $^{2}$ and Fares Sayegh $\mathbb{D}^{1}$ \\ ${ }^{1} 3^{\text {rd }}$ Orthopaedic Dept., Aristotle University of Thessaloniki Medical School, Papageorgiou General Hospital, Thessaloniki Ring \\ Road West, Nea Efkarpia, 56403 Thessaloniki, Greece \\ ${ }^{2}$ Department of Radiology, Papageorgiou General Hospital, Thessaloniki Ring Road West, Nea Efkarpia, 56403 Thessaloniki, Greece \\ ${ }^{3}$ Department of Pathology, Aristotle University of Thessaloniki Medical School, University Campus, 54124 Thessaloniki, Greece
}

Correspondence should be addressed to Kyriakos Papavasiliou; papavasiliou.kyriakos@gmail.com and Fares Sayegh; fsayegh@auth.gr

Received 3 April 2019; Revised 8 January 2020; Accepted 13 January 2020; Published 17 June 2020

Academic Editor: Josep M. Ribera

Copyright (C) 2020 Kyriakos Papavasiliou et al. This is an open access article distributed under the Creative Commons Attribution License, which permits unrestricted use, distribution, and reproduction in any medium, provided the original work is properly cited.

Only three cases of patients suffering from a localized chronic form of Langerhans cell histiocytosis (LCH) successfully treated with radiofrequency ablation (RFA) have been published so far. This is the first case report of a patient with a localized chronic form of LCH of the femur, which was successfully treated with percutaneous image-guided RFA, and who was evaluated pre-RFA and followed up post-RFA for a period of 48 months, in order to validate the safety and efficacy of this method and to obtain imaging studies depicting the actual in situ changes taking place post-RFA. RFA was proved to be a safe and efficient method when treating patients with a localized chronic form of LCH.

\section{Introduction}

Langerhans cell histiocytosis $(\mathrm{LCH})$ is a relatively rare disease of the musculoskeletal system $[1,2]$. Being more common in male than female patients, $\mathrm{LCH}$ is characterized by the existence of single or multiple skeletal lesions, occurring predominately in patients under twenty years of age and accounts for approximately $70 \%$ of the histiocytic neoplasms [3]. The localized chronic form of LCH is an even more rare form of the disease $[1,2]$. Several therapeutic methods for the treatment of $\mathrm{LCH}$ have been proposed and used with reported results of varying success [4].

The image-guided percutaneous thermoablation is a wellestablished treatment method for both the primary and the metastatic tumors of the musculoskeletal system. The goal of this procedure is to induce coagulation necrosis in the target tissue, by inserting special applicators in the tumor under image guidance. The lesion is heated to temperatures above $80^{\circ} \mathrm{C}$, usually with the use of the radiofrequency ablation (RFA) technique [5-7] [8]. The indications for RFA seem to continuously expand, mainly due to the significant advantages that this method has over surgical treatment $[6,7]$.

The treatment of the localized chronic form of $\mathrm{LCH}$ with the use of RFA was recently introduced in the English literature, and promising results have been reported following its implementation in three young patients $[9,10]$. The aim of this study is to present a new case of a patient with a localized chronic form of LCH in his right femur, who was successfully treated with RFA, and to present-to the best of our knowledge-for the first time, long-term imaging studies post- 


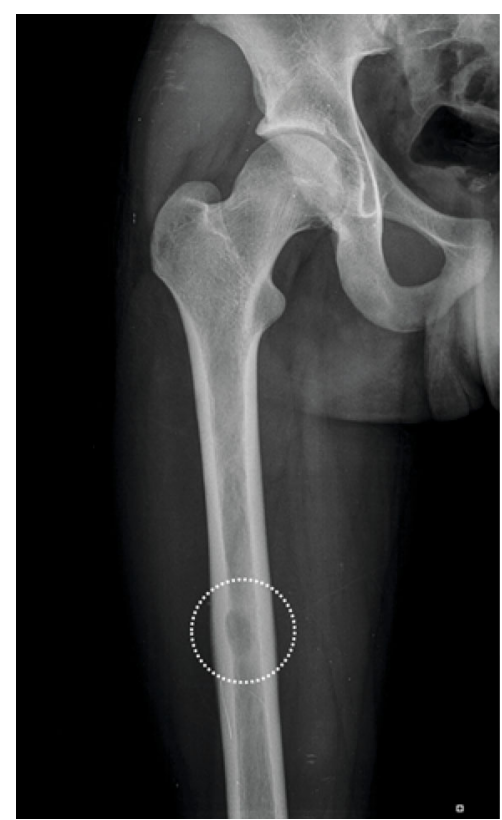

(a)

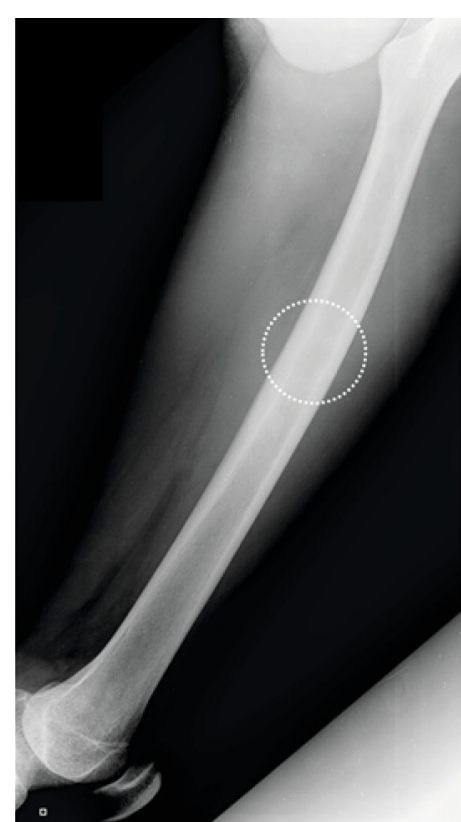

(b)

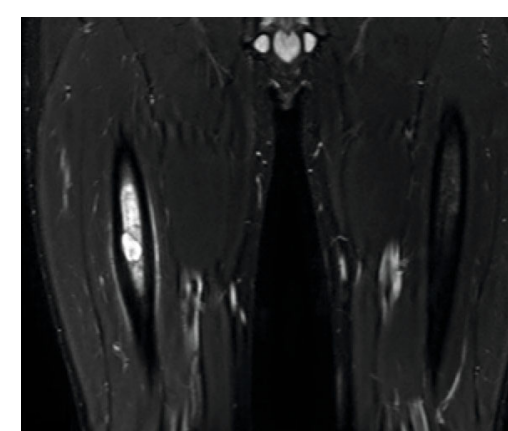

(c)

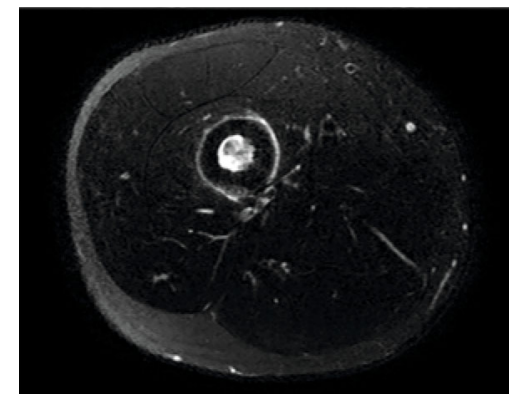

(d)

FIGURE 1: The initial plain radiograph of the femur ((a) anteroposterior and (b) lateral) depicting (dotted line) a centrally located lytic lesion at the femoral diaphysis with concomitant reactive cortical thickness. The initial MRI scan of the lesion. (c) Short T1 reverse- (STIR-) weighted image (WI) coronal view. (d) T2WI axial view (see text for more details).

RFA, validating and proving the safety and the efficacy of this novel method for the treatment of the localized chronic form of $\mathrm{LCH}$.

\section{Case Presentation}

An otherwise fit 16-year-old boy was referred for evaluation and treatment to our department (a tertiary Trauma and Orthopaedics center) by a general practitioner. The patient was suffering from pain on the lower right thigh with an onset of approximately four months before his initial evaluation. The pain was described as being initially dull and intermittent; later, it became continuous and significantly increased in severity. The pain was elicited on palpation at the affected area and aggravated by weight bearing. The ipsilateral hip and knee joints had a normal range of motion. Red and white blood cell count, C-reactive protein, erythrocyte sedimentation rate, biochemistry, and thyroid and parathyroid hormones levels, were all within normal range. Plain radiographs (Figures $1(\mathrm{a})$ and $1(\mathrm{~b})$ ) revealed the existence of a rounded and well-defined lytic lesion at the right femoral diaphysis with evidence of surrounding mild laminated chronic-type periosteal reaction. The patient subsequently underwent a CT and an MRI scan. CT scan revealed a $13 \times$ $24 \mathrm{~mm}$ eccentric lytic lesion with intact femoral cortices and no soft tissue involvement. The same lesion was depicted on the MRI scan (Figures 1(c) and 1(d)) as rounded and welldefined, with intermediate MR sign in the T1 and hyperintensity in the T2-weighted images. The lesion demonstrated intense but relatively homogenous enhancement after the intravenous administration of contrast. On the short $\mathrm{T} 1$ reverse- (STIR-) weighted images, intense surrounding bone oedema and a linear periosteal reaction were present.

The patient underwent core needle biopsy under general anesthesia and C-arm X-ray image intensifier control (in order to accurately locate radiographically the exact location of the lesion) with a $6 \mathrm{~mm}$ trocar. Culture swabs were also taken from the lesion area and came back as negative. The histopathologic study of the specimen showed a mixed cellular infiltrate made up of macrophages, lymphocytes, eosinophilic granulocytes, multinucleated cells, and Langerhans cells. Further, immunohistochemistry showed positive staining for S-100 protein and CD-1a antigen and confirmed the diagnosis of LCH. Further imaging studies (plain radiographs of the thoracic and lumbar spine, skull, and pelvis) and a $\mathrm{Tc}^{99}$ bone scan verified the unifocal form of the disease, and a bone marrow biopsy failed to reveal any other pathology.

Taking into consideration the solitary form of the disease, its location in a weight-bearing long bone, and the age of the patient and following consultation with a consultant radiologist, a pediatric-oncologist, and a pediatric-hematologist, we decided to perform RFA instead of open curettage or wide excision of the lesion followed by bone grafting and/or internal fixation.

The radiofrequency ablation procedure took place in the CT suite room under general anesthesia. A thin biopsy needle was initially introduced through the thickened femoral cortex via the track of the previously performed biopsy. A $15 \mathrm{~cm} / 11$-gauge RFA electrode needle (AMICA RF, HS Hospital Services S.p.A., Rome-Italy) was used. The RFA 


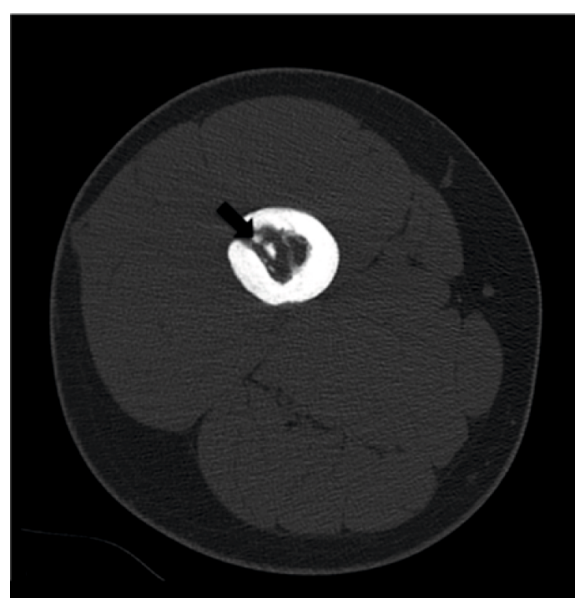

(a)

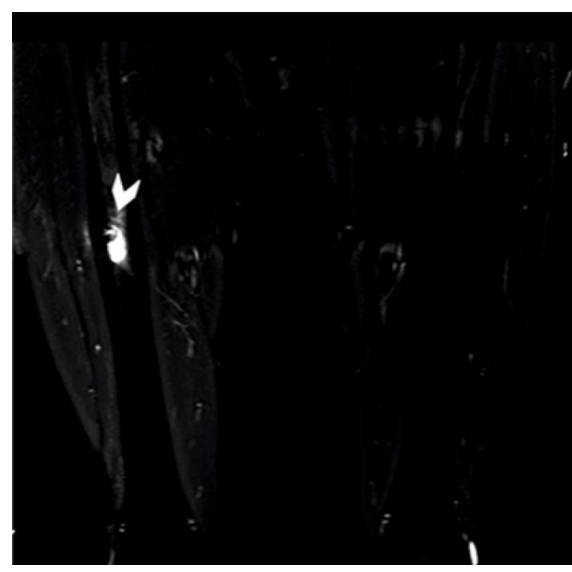

(c)

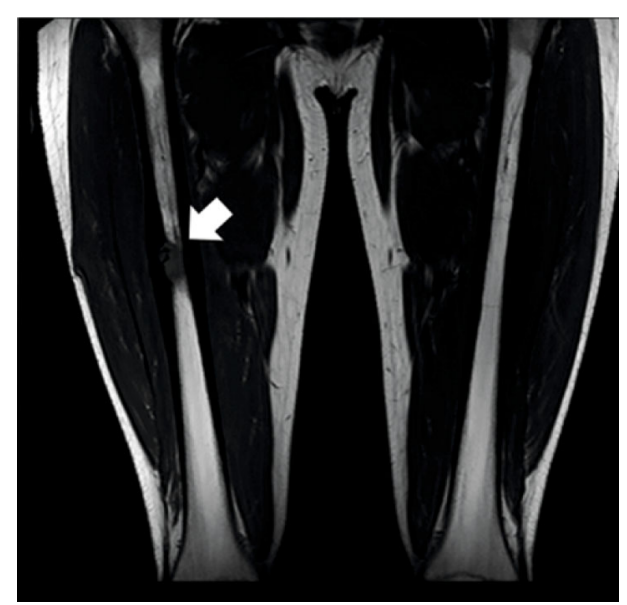

(b)

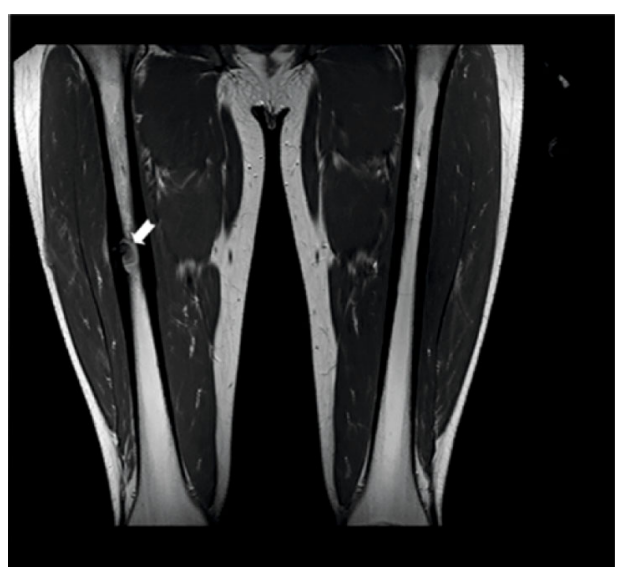

(d)

FIGURE 2: Axial CT image (a) at three months post-RFA, revealing the existence of dystrophic calcifications (black arrow). Coronal MRI scan images of the diaphysis of the right femur of the patient at three months post-RFA, depicting a well-defined lytic lesion with central necrosis. The necrosis had an intermediate to low MR sign on T1WI ((b), white arrow) and prominent surrounding oedema on STIR ((c), arrowhead). After the intravenous administration of contrast media, the lesion showed enhancement only at its periphery ((d) short arrow).

procedure was initiated following confirmation that the tip of the plain electrode was well placed in the center of the lesion. The electrode tip was heated up to $90-94^{\circ} \mathrm{C}$ for 8 minutes. After a cooling period of 1 minute, the electrode was safely retracted, followed by the probe and the cannula. The patient reported immediate relief from pain as soon as he woke up and was uneventfully discharged the day after. He was advised to walk without bearing weight and with the use of two crutches for one month.

At three months after the treatment, the patient was completely asymptomatic and able to walk with full weight bearing. The CT scan revealed a central zone with fluid density due to necrosis (Figure 2(a)). The MRI scan confirmed the central necrosis of the tumor, a moderate enhancement at the periphery of the lesion and a mild surrounding bone oedema, which was a consequence of the RFA procedure (Figures 2(b)-2(d)). At six months post-RFA, he reported no pain at all, he was capable of performing all physical activities, and he was completely happy with the overall result of his treatment. The CT scan depicted the existence of callus at the cortex hole area (Figure 3(a)). The MRI scan revealed a more prominent central necrosis, while the postcontrast enhancement and the surrounding bone oedema were decreased (Figures 3(b)-3(d)). During subsequent follow-up visits at $12,18,24$, and 36 months post-RFA, the MRI characteristics depicting necrosis at the RFA area gradually subsided, until they became normal at the last followup visit at month 48 (Figure 4). The patient remains still symptoms-free at his latest phone call follow-up visit six years post-RFA.

\section{Discussion}

The localized chronic form of Langerhans cell histiocytosis of the bone is a benign tumor-like condition, representing less than $1 \%$ of the tumor-like lesions of the bone $[11,12]$. About $90 \%$ of patients are between 5 and 15 years of age and there is a slight male predominance [11]. The bones which are the most commonly involved are the skull and the pelvis [13]. Over one-third of the lesions involve the long bones, with the femur being the most common site, followed by the humerus and tibia. Fibular involvement is rare. In the long 


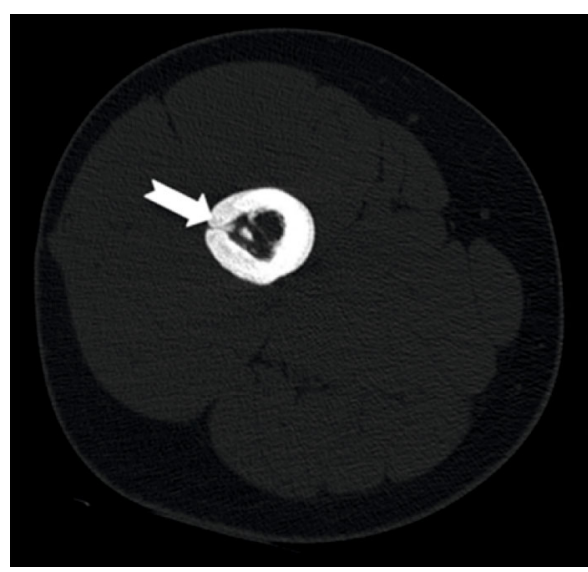

(a)

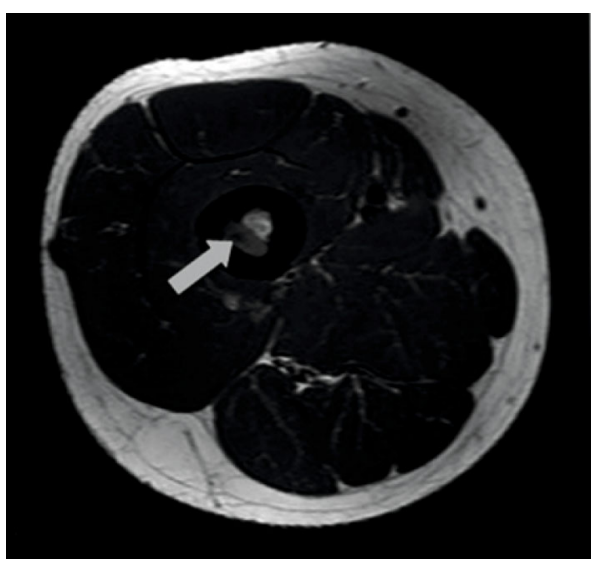

(c)

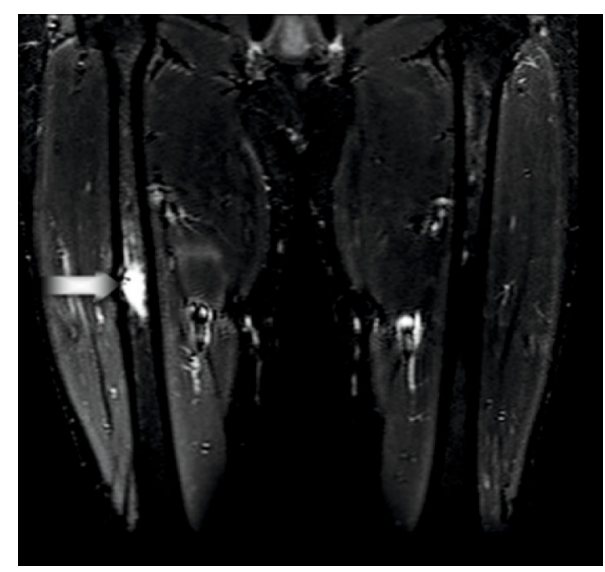

(b)

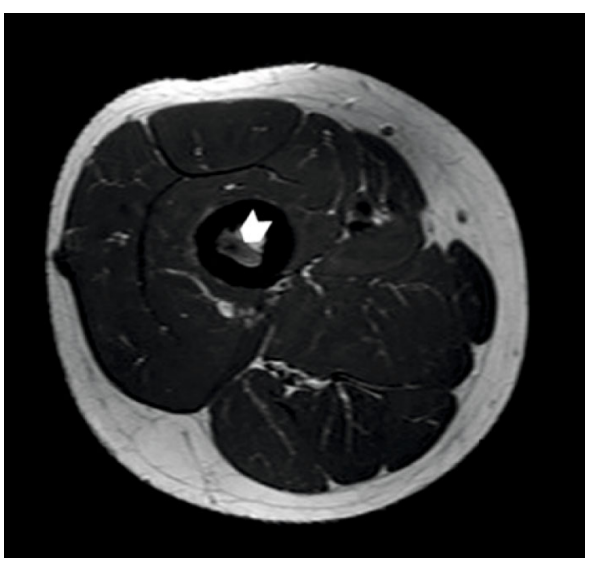

(d)

FIGURE 3: Axial CT scan (a) at six months post-RFA, depicting the formation of callous at the cortex hole area (white arrow). MRI scan at six months post-RFA. (b) Coronal STIR images showing decreased surrounding oedema (when compared with the previous scan) and necrotic appearance of almost the entire lesion (black and white arrow). Axial T1WI images before (c) and after (d) the administration of contrast media, depicting the central necrosis (grey arrow) and the peripheral type of enhancement (short arrow).

bones, the diaphysis is most commonly affected, followed by the metaphysis and rarely the epiphysis [14].

The primary target when treating patients with the localized chronic form of $\mathrm{LCH}$ is to achieve complete relief from pain, to prevent pathological fracture and growth disturbances, and to allow returning of the patient to his/her normal everyday-life activities [2, 4, 9]. Several forms of treatment have been proposed, ranging from the simple observation to significantly more invasive ones [13]. The treatment of choice seems to be wide excision and/or curettage, followed by bone grafting [9]. However, operative treatment in particular, although it seems to be rather successful in terms of symptom resolution and low recurrence rates, it may be accompanied by increased morbidity and complications. Alternatively, and in search of a less invasive treatment method, the intralesional instillation of steroids and the external radiation have also been tried, with varying success rates [9]. Complete resolution of the symptoms is reported in $92 \%$ and complete imaging resolution in $95 \%$ of the patients following the intralesional injection of methylprednisolone [15]. Chemotherapy and external beam radiation have also been reported to have very good results, with a recurrence rate of less than $20 \%$ [13]. However, chemotherapy is not easily accepted by a patient for a benign condition and both treatment modalities may lead to the later development of severe complications.

Radiofrequency ablation under radiographic guidance is a well-established and easy-to-perform method of treatment for several benign and malignant bone lesions [5]. This method offers immediate pain relief, shorter rehabilitation period for the patient, brief hospitalization, less cost, less soft tissue damage, and minimal blood loss [16, 17]. Due to all these advantages and the fact that its use does not necessitate the purchase of expensive equipment, it has gained worldwide acceptance and recognition. Its use in the treatment of patients suffering from $\mathrm{LCH}$ has been recently introduced and the results reported so far in three patients are very promising $[9,10]$.

We report-to the best of our knowledge-the fourth case in the English literature of a patient suffering from a localized chronic form of $\mathrm{LCH}$ who was successfully treated with RFA (Table 1). The patient experienced immediate relief from his symptoms, suggesting adequate and successful treatment. Furthermore, the imaging studies obtained 


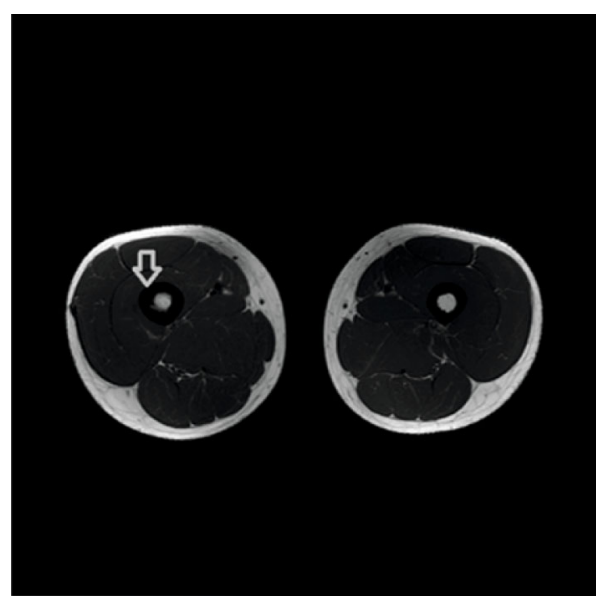

(a)

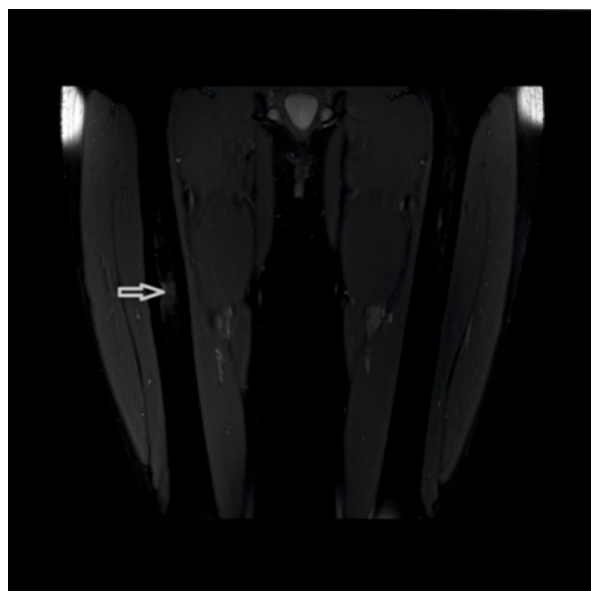

(c)

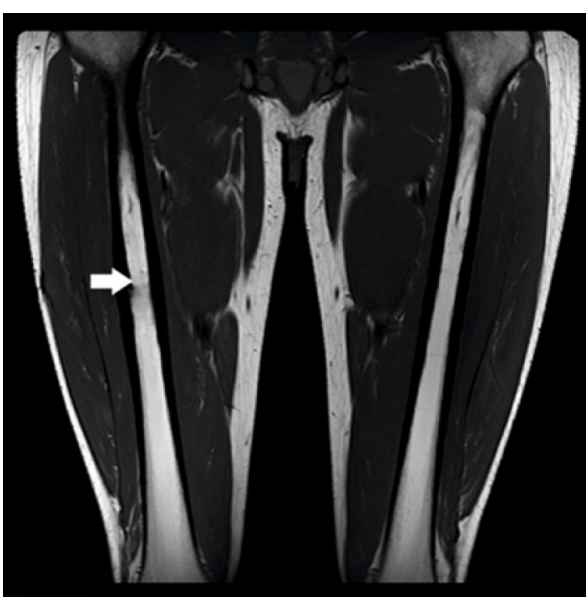

(b)

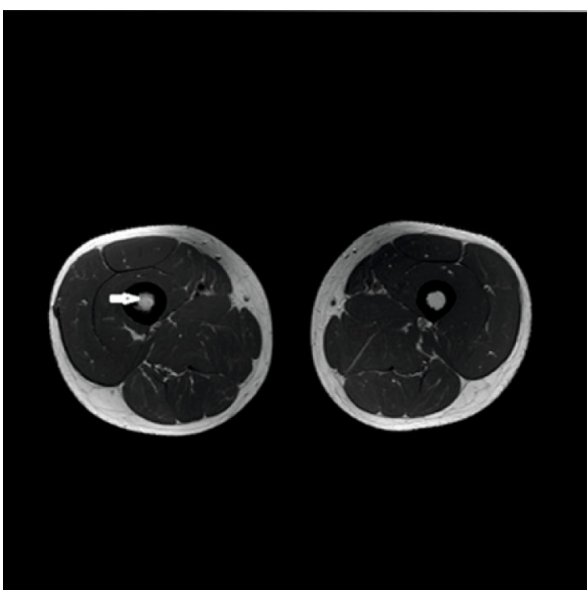

(d)

FIgURE 4: MRI scan at 48 months post-RFA. Axial (a) and coronal (b) T1WI images show no bone oedema (white arrow) and only minor cortical thickening (arrowhead). Proton density WI (PDWI) with fat suppression MRI scan image (c) verified the absence of bone oedema (grey arrow). Axial T1WI MRI scan image (d) following the administration of contrast media suppression show no enhancement.

TABLE 1: Summary of the previously reported patients' characteristics and outcomes and comparison with this study's data. M: male, F: female, RFA: radiofrequency ablation.

\begin{tabular}{|c|c|c|c|c|c|c|}
\hline Study & Age & Sex & Location of the lesion & Outcome & Latest follow-up & Imaging modality used \\
\hline \multirow{2}{*}{ Corby et al. } & 7 & $\mathrm{~F}$ & Femoral diaphysis & $\begin{array}{c}\text { Full resolution of symptoms } \\
7 \text { days post-RFA }\end{array}$ & 12 months post-RFA & Radiograph \\
\hline & 14 & $\mathrm{~F}$ & Iliac & $\begin{array}{l}\text { Full resolution of symptoms } \\
2 \text { days post-RFA }\end{array}$ & 11 months post-RFA & Radiograph \\
\hline Beccee et al. & 18 & M & Iliac & Full resolution of symptoms & 12 months post-RFA & N/A \\
\hline Present study & 16 & $\mathrm{M}$ & Femoral diaphysis & $\begin{array}{l}\text { Full resolution of symptoms } \\
\text { immediately post-RFA }\end{array}$ & 48 months post-RFA & Radiograph, CT scan, MRI scan \\
\hline
\end{tabular}

following the RFA prove beyond any doubt the therapeutic value of this novel method of treatment, by clearly depicting the complete necrosis of the lesional area and the subsequent regeneration of normal tissue during the following 48 months.

Even though this is a case report, there are some limitations that must be mentioned. One is that the patient could have been successfully treated with alternative therapeutic methods as well, even with a simple observation. However, he refused to do so because he could not tolerate the pain and he wanted a permanent and rapid treatment for his condition. PET CT, which is also a valuable modality in the primary assessment, therapeutic monitoring, and detection of reactivation of patients with $\mathrm{LCH}$, could also have been used as a means to evaluate the efficacy of the RFA procedure during the follow-up evaluations. However, the increased 
cost and the dosimetric considerations for ionizing radiation uptake were the reasons we chose the MRI scans instead.

Radiofrequency ablation, as a novel method of treatment for the localized chronic form of $\mathrm{LCH}$, certainly needs to be further validated by a large series of patients. Given however the rarity of the disease, the previously reported and effectively treated three patients, the hereby reported patient, and the reduced morbidity rates accompanying this method, it seems that RFA is a highly successful method of treatment when dealing with patients suffering from a localized chronic form of Langerhans cell histiocytosis.

\section{Conflicts of Interest}

The authors declare that the companies HS Hospital Services S.p.A. (Rome-Italy) and Impact Vogdaneris Ltd. (AthensGreece), partially covered the article processing charges of this publication.

\section{References}

[1] J. C. Leonidas, M. Guelfguat, and E. Valderrama, "Langerhans' cell histiocytosis," Lancet, vol. 361, no. 9365, pp. 1293-1295, 2003.

[2] M. Kobayashi and A. Tojo, "Langerhans cell histiocytosis in adults: advances in pathophysiology and treatment," Cancer Science, vol. 109, no. 12, pp. 3707-3713, 2018.

[3] P. J. Papagelopoulos, A. F. Mavrogenis, E. C. Galanis et al., "Minimally invasive techniques in orthopedic oncology: radiofrequency and laser thermal ablation," Orthopedics, vol. 28, no. 6, pp. 563-568, 2005.

[4] S. Noordin, S. Allana, K. Hilal et al., "Osteoid osteoma: contemporary management,” Orthop Rev (Pavia), vol. 10, no. 3, p. 7496, 2018.

[5] A. Angelini, A. F. Mavrogenis, E. Rimondi, G. Rossi, and P. Ruggieri, "Current concepts for the diagnosis and management of eosinophilic granuloma of bone," Journal of Orthopaedics and Traumatology, vol. 18, no. 2, pp. 83-90, 2017.

[6] K. Ringe, M. Panzica, and C. von Falck, "Thermoablation of bone tumors," RöFo-Fortschritte auf dem Gebiet der Röntgenstrahlen und der bildgebenden Verfahren, vol. 188, no. 6, pp. 539-550, 2016.

[7] A. S. Haouimi, Z. M. Al-Hawsawi, and A. N. Jameel, "Unusual location of eosinophilic granuloma," Saudi Medical Journal, vol. 25, no. 10, pp. 1489-1491, 2004.

[8] R. R. Corby, G. S. Stacy, T. D. Peabody, and L. B. Dixon, "Radiofrequency ablation of solitary eosinophilic granuloma of bone," American Journal of Roentgenology, vol. 190, no. 6, pp. 1492-1494, 2008.

[9] F. Becce, N. Theumann, A. Rochette et al., "Osteoid osteoma and osteoid osteoma-mimicking lesions: biopsy findings, distinctive MDCT features and treatment by radiofrequency ablation," European Radiology, vol. 20, no. 10, pp. 2439-2446, 2010.

[10] A. Eroglu, I. C. Kürkçüoglu, and N. Karaoglanoglu, "Solitary eosinophilic granuloma of sternum," The Annals of Thoracic Surgery, vol. 77, no. 1, pp. 329-331, 2004.

[11] J. F. Emile, O. Abla, S. Fraitag et al., "Revised classification of histiocytoses and neoplasms of the macrophage-dendritic cell lineages," Blood, vol. 127, no. 22, pp. 2672-2681, 2016.
[12] F. Plasschaert, C. Craig, R. Bell, W. G. Cole, J. S. Wunder, and B. A. Alman, "Eosinophilic granuloma," Journal of Bone and Joint Surgery. British Volume (London), vol. 84-B, no. 6, pp. 870-872, 2002.

[13] E. M. Azouz, G. Saigal, M. M. Rodriguez, and A. Podda, "Langerhans' cell histiocytosis: pathology, imaging and treatment of skeletal involvement," Pediatric Radiology, vol. 35, no. 2, pp. 103-115, 2005.

[14] A. F. Mavrogenis, C. N. Abati, G. Bosco, and P. Ruggieri, "Intralesional methylprednisolone for painful solitary eosinophilic granuloma of the appendicular skeleton in children," Journal of Pediatric Orthopedics, vol. 32, no. 4, pp. 416-422, 2012.

[15] P. S. Yip, Y. L. Lam, M. K. Chan, J. S. Shu, K. C. Lai, and Y. C. So, "Computed tomography-guided percutaneous radiofrequency ablation of osteoid osteoma: local experience," Hong Kong Medical Journal, vol. 12, no. 4, pp. 305-309, 2006.

[16] O. Ofluoglu, "Minimally invasive management of spinal metastases," The Orthopedic Clinics of North America, vol. 40, no. 1, pp. 155-168, 2009.

[17] J. Freebody, E. A. Wegner, and M. A. Rossleigh, “2-deoxy-2((18) F)fluoro-D-glucose positron emission tomography/computed tomography imaging in paediatric oncology," World Journal of Radiology, vol. 6, no. 10, pp. 741-755, 2014. 